\title{
EXPERIMENTAL INVESTIGATION OF CYLINDRICAL FOOD PRODUCT DURING FORCED CONVECTION COOLING
}

\author{
Taliv Hussain*, Zafar Alam and Adnan Hafiz \\ Mechanical Engineering Department, Aligarh Muslim University, Aligarh, India.202002 \\ * Author corresponding Email:hussaintaliv@gmail.com
}

\begin{abstract}
The objective of this work is to study the transient heat transfer behavior during precooling of cylindrical food product at different air flow rates. Temperature measurement is carried out at the center of the specimen at different air flow rates (3.8, 4.0, $4.2 \& 4.5 \mathrm{~m} / \mathrm{s}$ ). These temperature distributions are plotted with time. The terms of Fourier equation were evaluated and thermal diffusivity was plotted against the temperature variation during cooling of the cylindrical food product.Surface film conductance values is also calculated in present work, at different air flow rates. These values are effective values, which included the additional cooling effect due to moisture evaporation from the exposed product surface. Variation of Reynolds number and Nusselt number is also plotted with different air velocities and it is found that more favorable results of thermal conductivity and thermal diffusivity is obtain at $3.8 \mathrm{~m} / \mathrm{s}$. Which ultimately saves energy and provide optimum cooling for the specimen at the given air velocity.
\end{abstract}

Keywords:Forced convection, transient heat transfer, cooling, thermal diffusivity, thermal conductivity

\section{INTRODUCTION}

The temperature of cultivation items is quite possibly the main parameterthat oversee the postharvest life and nature of particular item. Precooling alludes to quick evacuation of field heat not long after gather of harvest. Field warmth can be characterized as distinction in temperature between the temperature of yield gathered and ideal stockpiling temperature of that item. By and large the temperature ought to be chilled off till it arrives at $88 \%$ of current contrast in temperature and its ideal stockpiling temperature[1]. Field warmth ought to be eliminated as quick as conceivable, since for most produce an hour delay at field states of about $35^{\circ} \mathrm{C}$ will prompt a misfortune in timeframe of realistic usability of around 1 day even at ideal stockpiling conditions. By the by, because of natural factors, the significance of quick pre-cooling fluctuates. As per Indian Board of Horticulture, particularly grapes,berries, mandarins,leeches, cherries, 
melons, stone organic products, okra, capsicum, tomatoes, stew peppers, cucumbers,peas, green beans and spinach ought to be quickly pre-cooled, while other less transitory produce is comprised of mangoes,pomegranates, papaya, guava, radish, cabbage, cauliflower,green bananas and carrots. There are a few techniques that can be picked to pre-cool produce [2].Nicholas and Plug(1962)[3]studied whitening of new, entire cucumbers is a typical business practice to build acridity of cucumbers to encourage their pressing into containers. The impact of whitening on nature of new cucumber pickles has been contemplated. Murthy et al. (1976)[4] studied heat move attributes during air cooling of clammy food items are dissected by considering the impacts of the vanishing of the dampness film that generally exists on the item surface. The one dimensional transient warmth conduction condition in round and tube shaped directions is settled. Majeed (1982) [5] presented anticipated cooling qualities during air cooling of barrel shaped food items with a streaming film of cold water atsurface of air-film cooling.Michelis andCalvelo(1983) [6]studied a improved model recently created by for freezing time estimations in plate coolers is reached out to frameworks with a few dimensional warmth stream. The model consolidates Plank's condition with the temperamental warmth move answers for bodies with consistent properties, through the expansion of precooling, change of stage and treating times. Ansari and Afaq(1986) [7] studied a computation conspire has recently been accounted for by for foreseeing air shoot cooling of circular food items. This depended on the perception that the cooling impact of item drying up is critical during the underlying stages yet gets irrelevant later. This work was expanded with the goal that the estimation plan can likewise be utilized for tube shaped items. Another PC condition is utilized for the psychometric properties of air. Chung and Merritt (1991) [8]presented a model which was created by comprising of an adjusted Plank's condition to appraise stage change time and two temperamental state cross-product heat move conditions for assessing precooling and treating times. It precisely anticipated absolute opportunity to continue from an underlying temperature over the edge of freezing over to a last temperature of $-18^{\circ} \mathrm{C}$.Dincer and Dost (1996) [9] studied precooling is a basic stage in the virus chain. During precooling cycle, field temperature offood item is brought down to capacity temperature promptly, after gather, to forestall any food decay and to keep up nature of products of the soil. Sadashive et al. (1996) [10] investigated the warmth move rates delivered by various cooling strategies given by can be straightforwardly acquired from the time-temperature variety bends of the item. In the event that the math, thermo-physical properties and preparing states of 
food item are known, time-temperature varieties can be hypothetically decided.Fasina and Fleming (2001) [11] studied the conceivable utilization of whitening in mix with controlled maturation is being considered as a way to lessen salt levels required in capacity of tenderized cucumbers. To be monetarily achievable, utilization of warmth ought to be enhanced for monetary and item quality contemplations. This essential data on the warmth move qualities of cucumbers expected to advance the whitening cycle.Brosnan and Sun (2001) [12] have recommended that it is fundamental not exclusively to cool the item yet additionally to cool item as fast as conceivable after reap. During pre-cooling, transient warmth move between liquid medium and strong food item takes place.Wu et al. 2004 [13] presented that there are a few articles in writing depicting drying of rural items utilizing limited volume strategy. Lima (2005) [14] presented that not many works utilize limited volume technique to depict cooling of items, especially of an individual strong, in spite of the way that this strategy presents a basic actual translation of the preservation laws.Erdogdu(2005) [15] studied that the warm diffusivity $\alpha$ and convective warmth move coefficient $\mathrm{h}$ are significant boundaries to describe, to demonstrate and to upgrade the warmth move measures.To decide these boundaries and to portray the cooling energy of an item, in an overall way two instruments are accessible: the scientific and mathematical apparatuses. Erdogdu (2008) [16]the synchronous assurance of warm diffusivity and convective warmth move coefficient is hard to achieve on the grounds that there might be a few sets of these boundaries for which the arrangement of the dispersion condition is fitted to a bunch of test information. At the end of the day, the target capacity may have a few nearby minima. Accordingly, the concurrent assurance of the boundaries of the dispersion condition through enhancement is an issue which actually merits further investigation.kumar et al. (2008) [17] studied a test examination has been done to consider the warmth move during constrained air precooling of tomato and orange at various cooling speeds of cold air at $4-5{ }^{\circ} \mathrm{C}$. Air speed has been fluctuated from 1.2 to $4.4 \mathrm{~m} / \mathrm{s}$. It was discovered that air speed had huge bearing on cooling paces of food items beneath the dimensionless temperature $(\mathrm{T})$ of 0.6 . The restricting cooling air speed for orange was $3.5 \mathrm{~m} / \mathrm{s}$ and that for tomato was $2.6 \mathrm{~m} / \mathrm{s}$. Silva et al. (2011) [18]investigated best model in the portrayal of cucumber's shape was ellipsoid, yet time requested in its improvement was around multiple times more noteworthy than ideal opportunity for boundless cylinder.Cooling of products of the soil, following the reap, has been a generally utilized strategy for boosting post-collect life. Silva and Silva(2014) [19] studied an numerical 
solution and an optimization algorithm which was used to determine simultaneously the thermal diffusivity $(\alpha)$ and the convective heat transfer coefficient (h) for an individual solid with cylindrical shape by using experimental data obtained during its cooling.

In this research paper, the main objective is to study the transient heat transfer behavior during precooling of cylindrical food product at different air flow rates. Temperature measurement is carried out at the center of the specimen (cucumber) at different air flow rates (3.8, 4.0, 4.2 \& 4.5 $\mathrm{m} / \mathrm{s})$.

\section{EXPERIMENTAL SETUP AND PROCEDURE}

Experiments were carried out on cylindrical shaped food sample. Experimental measurement of transient time temperature behaviour during precooling of the food product is done, with approximately cylindrical geometry in forced cooling environment (Fig 1).

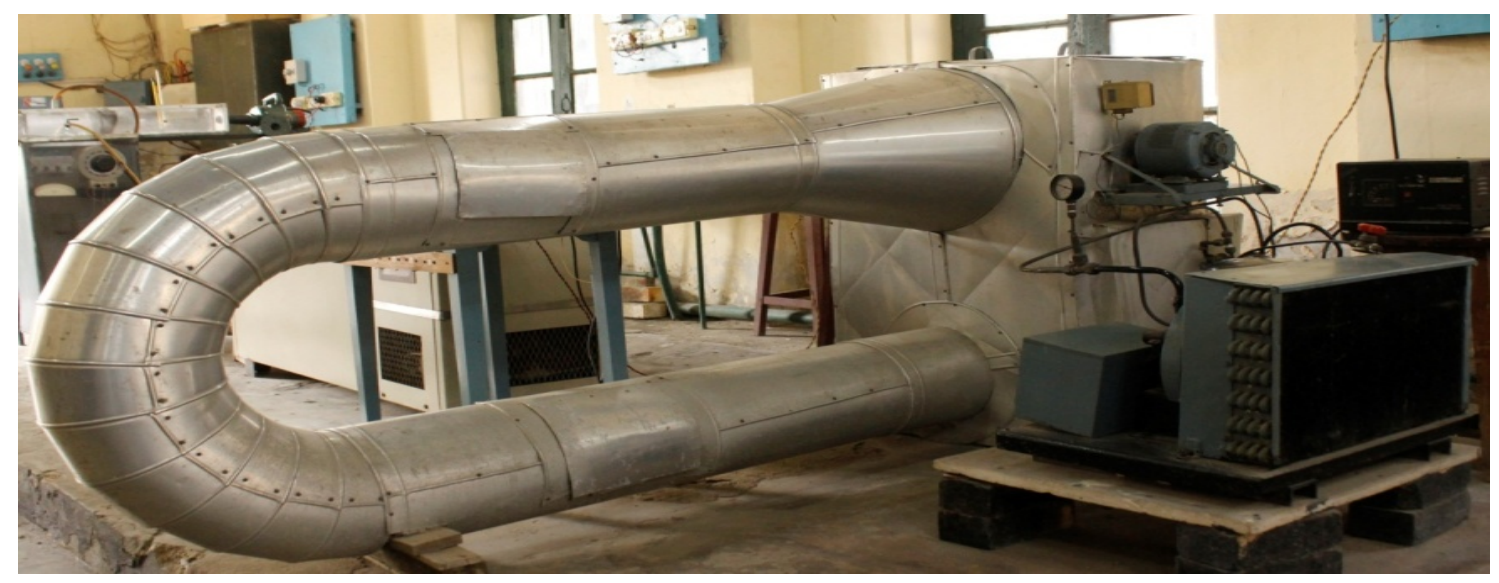

Fig 1.Line diagram of U-Duct for forced convection cooling

\subsection{Specification of the setup}

The forced convection apparatus comprises of a chilled air duct with vapour compression refrigeration system, microvolt ammeter and air blower, anemometer, copper constantan thermocouple.

1) $1.06 \mathrm{~m} \times 0.6 \mathrm{~m} \times 0.4 \mathrm{~m}$ refrigerated fortification made of $22 \mathrm{~S}$.W.G aroused iron sheet with a $0.4 \mathrm{~m}$ breadth opening at the top and a $0.2 \mathrm{~m}$ measurement opening at the base end.

2) Refrigerant used in the setup is R-22. 
3) Rotary compressor with 1-ton capacity, Fined-Tube type air-cooled condenser with fan,fins and Copper tube type cooling coil.

4) From the upper opening a tightened conduit $0.45 \mathrm{~m}$ since quite a while ago was joined and its opposite end with a $0.2 \mathrm{~m}$ breadth shut channel. The entire conduit was then protected by around $3 \mathrm{~cm}$ thick glass fleece.

5) Blower was used for air circulation. Air speed inside the duct can be change using a voltage regulator. Air was recirculated again and again across cooling coils of refrigeration system.

\subsection{Instruments Used}

In order to obtain required data of time temperature measurements, necessaryinstruments used are as follows:

1) Anemometer- In the cooling duct,air stream velocity was measured by an anemometer.

2) Vernier Calliper- Diameter of the food samples were measured by vernier calliper with a least count of $0.02 \mathrm{~mm}$.

3) Stop watch- The time interval was measured by the stop watch.

4) Thermocouple- temperature measurement is done bythe copper constantan thermocouple which is linked to a microvolt ammeter.

\subsection{Standardization of thermocouple}

Copper-constantan thermocouples utilized for temperature estimation were aligned subsequent to interfacing them to Digital DC microvolt ammeter. A mercury thermometer of reach 0 to $100{ }^{\circ} \mathrm{C}$ and least tally of $0.1^{\circ} \mathrm{C}$ were utilized as standard. Thermocouple intersection was fixed in contact with mercury bulb of thermometer. This System was then submerged in a jar of squashed ice. Both thermocouple and the thermometer showed a similar temperature (inside $\pm 0.1^{\circ} \mathrm{C}$ ) . To change over the millivolts estimated by advanced DC microvolt ammeter to ${ }^{\circ} \mathrm{C}$, the accompanying recipe is utilized:

\section{$\mathrm{C}=0.011372033+25.945621 \times \mathrm{MV}-0.70318373 \times \llbracket \mathrm{MV} \rrbracket \wedge 2$}

Where, C symbolizes Temperature in degree centigrade and MV is the digital DC microvolt ammeter reading. 


\section{Methodology}

In forced convection cooling, we first run the vapour compression refrigeration system and air blower in the U-duct until a steady low temperature is reached inside the duct. The sample was suspended with the help of specially fabricated frame and hook in the test section. Copper Constantan Thermocouple was used to measure temperature of product. Thermocouple were inserted in food product at center. The temperature was measured until the temperature approaches the temperature of cooling medium with an interval of one minutes. The time and temperature were recorded for cylindrical food product cucumber. The temperature was measured in degree Celsius with $\mathrm{T}$ type thermocouples connected to a microvolt ammeter. The characteristics dimensions of the cylindrical food product is measured by using a vernier caliper of least count $0.02 \mathrm{~mm}$. Fig. 2 shows the food sample (cucumber). Measured dimensions are as follows:

Length-156.73mm

Diameter- 34.02mm

$\mathrm{W}=0.96[20]$

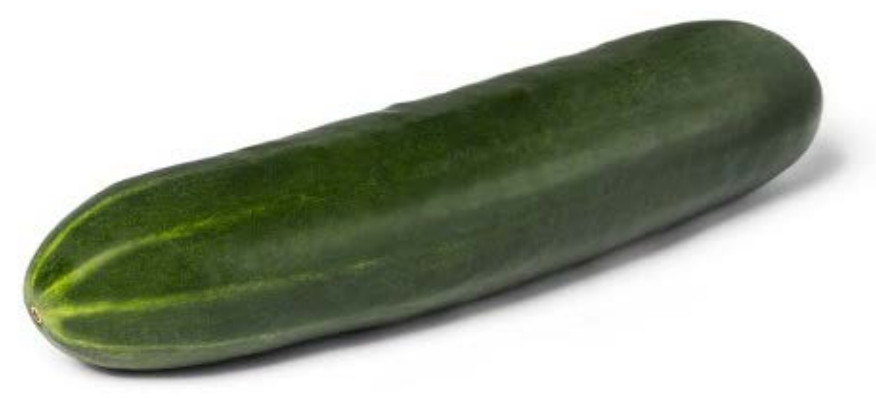

FIG.2Food sample (cucumber)

\section{Governing Equations}

For a produce which can be approximated with a limitless chamber and which is isotropic, at a uniform beginning temperature and is cooled evenly, the standardized transient warmth 
conduction condition, introductory condition and limit condition along the focal hub are, individually

$\frac{\partial^{2} \mathrm{U}}{\partial x^{2}}+\frac{1}{R} \times \frac{\partial \mathrm{U}}{\partial \mathrm{R}}=\frac{\partial \mathrm{U}}{\partial \tau} \quad$ for $\quad 0 \leq \mathrm{R} \leq 1, \tau>\tau_{i}(1)$

$\mathrm{U}=1.0 \quad$ for $0 \leq \mathrm{R} \leq 1, \tau=\tau_{i}$

$\partial \mathrm{U} / \partial \mathrm{R}=0$

for $\quad \mathrm{R}=0, \quad \tau>\tau_{i}$

$\partial \mathrm{U} / \partial \mathrm{R}=-B_{i} U$ for $\quad \mathrm{R}=1, \quad \tau>\tau_{i}$

$\partial \mathrm{U} / \partial \mathrm{R}=-B_{i}\left(C_{1} U^{2}+C_{2} U+C_{3}\right)$ for $\quad \mathrm{R}=1, \quad \tau>\tau_{i}$

On the off chance that no dampness vanishing happens from the produce surface, its warmth misfortune will be an element of the temperature contrast between the produce surface and the encompassing medium. For this situation, the surface limit condition will be characterized by the Newton's law of cooling as given above by Equation (4). In the event that dampness dissipation is happening from the produce surface, its absolute warmth misfortune will be a component of enthalpy potential, which exists between item surface and cooling air and is given above, by Equation (5). The necessary surface film conductance was determined from the $\mathrm{Nu} \mathrm{Re}$, relationship accessible in the writing for normal shape bodies are given as-

$\mathrm{Nu}=\mathrm{p}+\mathrm{q} \cdot \operatorname{Re}^{\mathrm{r}} \cdot \mathrm{Pr}^{\mathrm{s}}$

p,q,r and s are the constants in the equation (6) are dissimilar for unlike shapes. These constants values are given in Table 1, given below:

TABLE 1

CONSTANTS IN EQUATION(6)

\begin{tabular}{|c|c|}
\hline Constant & Infinite cylinder \\
\hline$p$ & 0.0 \\
\hline$q$ & 0.26 \\
\hline$r$ & 0.6 \\
\hline$s$ & -0.300 \\
\hline
\end{tabular}




\section{Formulae used}

\subsection{Determination of thermo-physical Properties}

Thermal diffusivity can calculated by the following empirical co relation stated by Gaffeny et al. [21]:

$$
\tau=[\mathrm{a}+\mathrm{b} \cdot \ln (1 / \mathrm{X}+0.2)-\ln \mathrm{U}] /\left[\mathrm{c} /\left(1+\mathrm{d} / B i^{e}\right)\right.
$$

TABLE.2

\section{CONSTANTS (REGRESSION) IN EQUATION (7)}

\begin{tabular}{|c|c|}
\hline CONSTANT & INFINITE CYLINDER \\
\hline $\mathrm{a}$ & 0.121 \\
\hline $\mathrm{b}$ & 0.201 \\
\hline $\mathrm{c}$ & 5.783 \\
\hline $\mathrm{d}$ & 2.45 \\
\hline $\mathrm{e}$ & 1.040 \\
\hline
\end{tabular}

From the known water content,the thermal conductivity of food product is determined using the following empirical co relation given by Mascheroni [22]:

$\mathrm{k}=0.1075+0.502 \mathrm{~W}+5.052 * 10^{-4} \mathrm{~W} . \mathrm{T}$

Where $\mathrm{W}$ is product water content on \% mass basis.

\subsection{Assumptions}

- Infinite Cylinder is assumed i.e. $\mathrm{R} \ll \mathrm{L}$, here R (Radius) And L (Height ofCylinder).

- The Solid assumed IsotropicandHomogeneous.

- The Spatial Dispersal of The Temperature isUniform and Have Radial Symmetry.

- Transport Of Heat in Solid Is only by Diffusion.

- During Heat diffusion,Convective Coefficient of Heat Transfer is Constant.

- Temperature of Cooling Mediumis Constant.

- During The Process, No Change ofPhase inthe Product.

- Owing To the Respiration,Mass Loss and Source Term.

- During The Cooling Of The CucumberHeat Are Insignificant. 


\section{Results and Discussion}

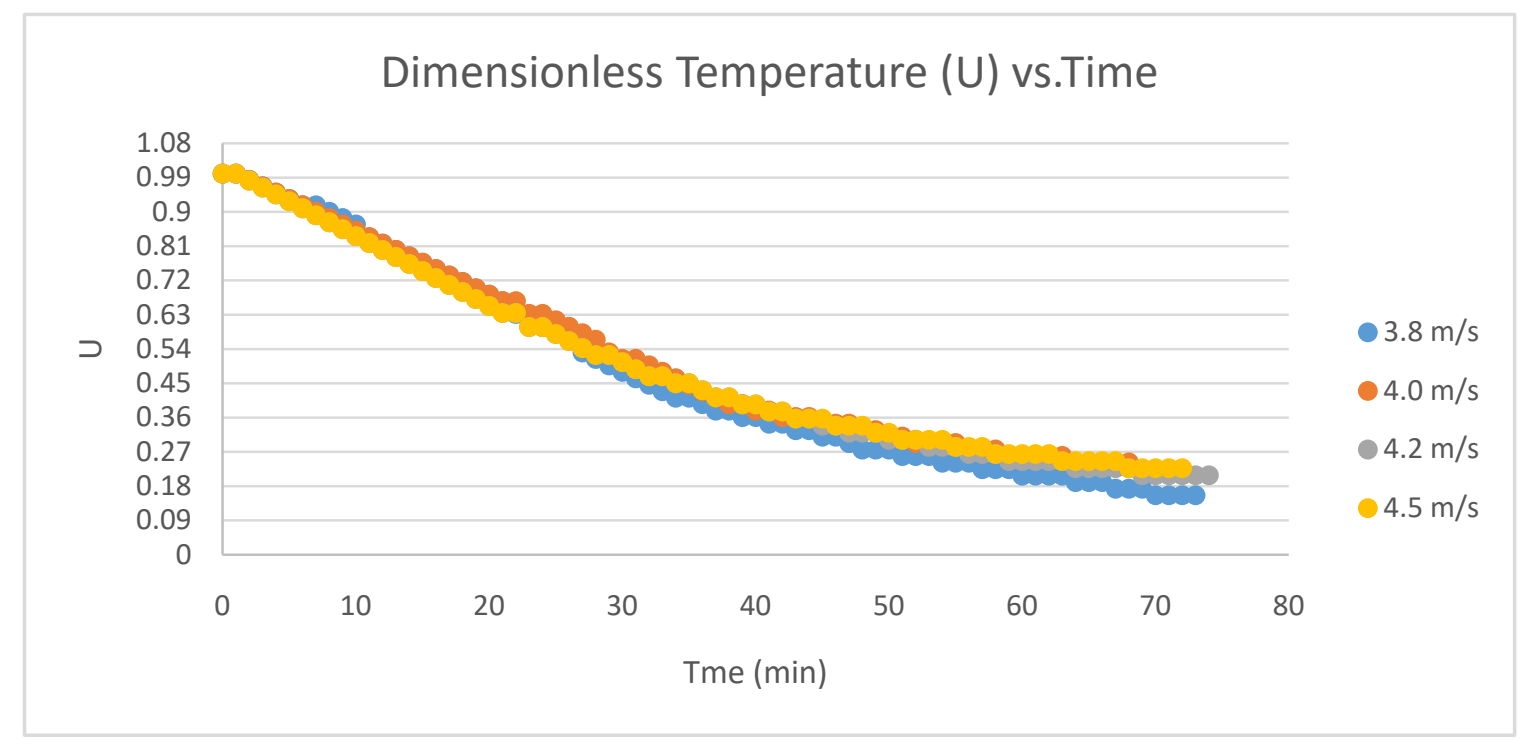

FIG.3 Variation of Dimensionless Temperature U with Time (min)

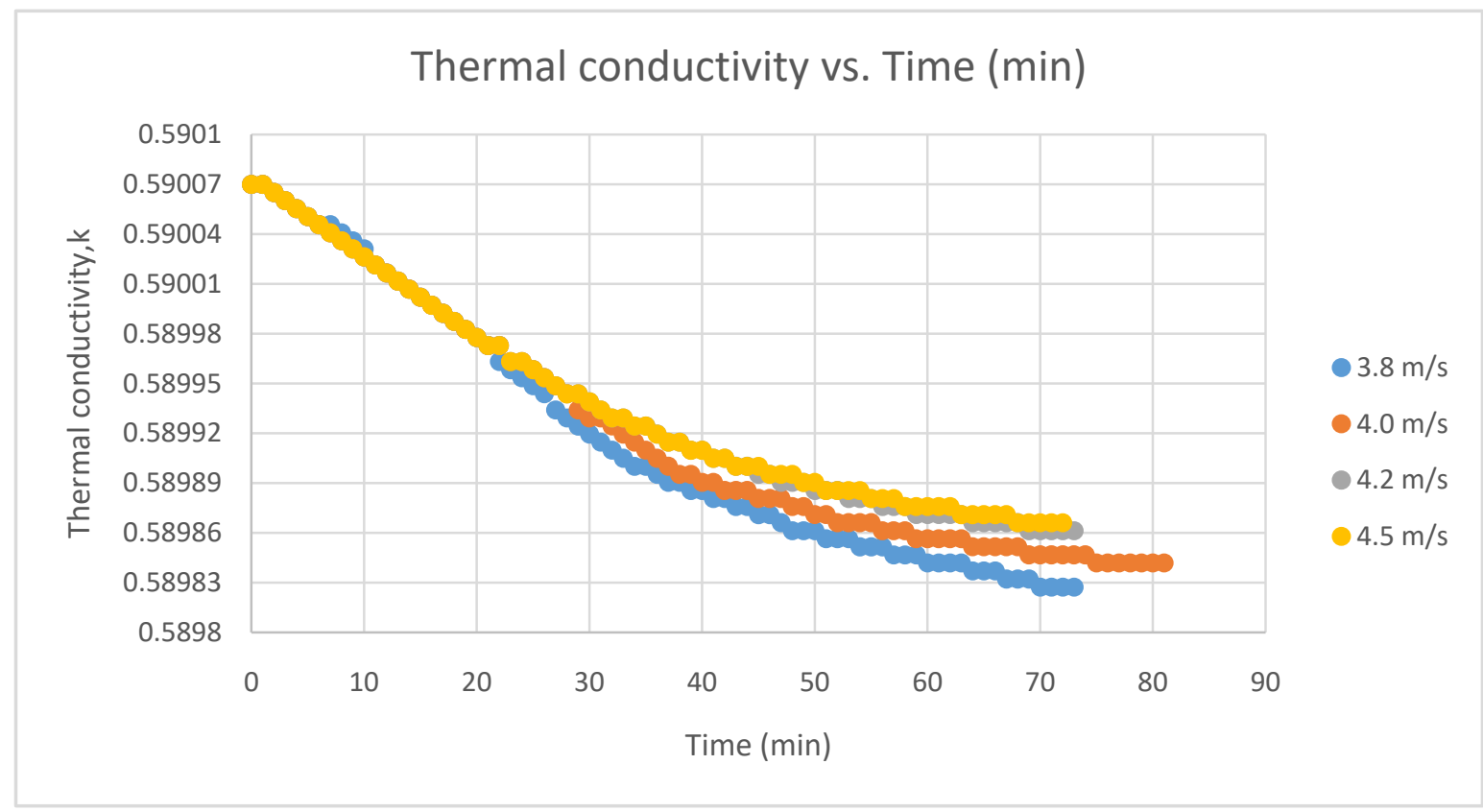

FIG.4 Variation of thermal conductivity, k with time (min) 


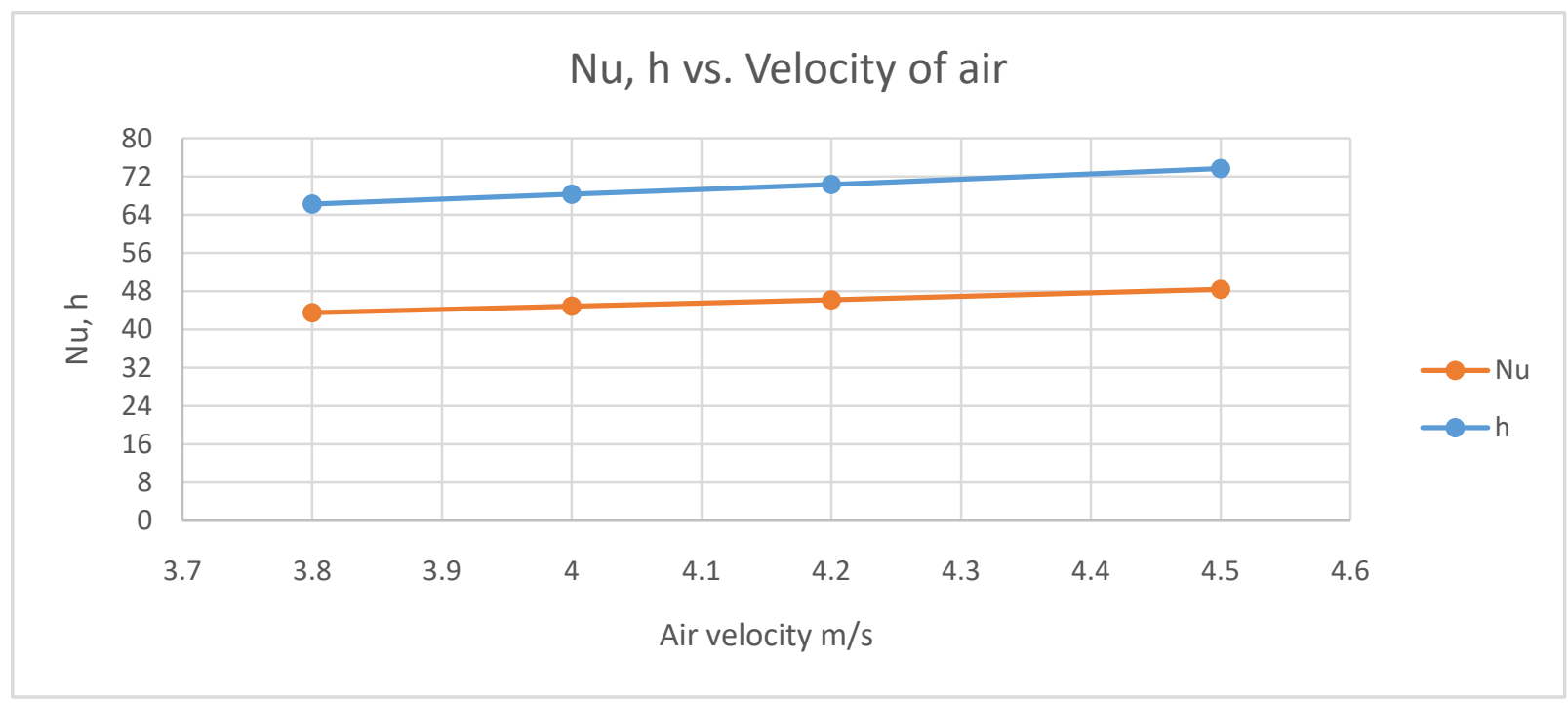

FIG.5 Variation of Nusselt No. and surface film conductance with velocity

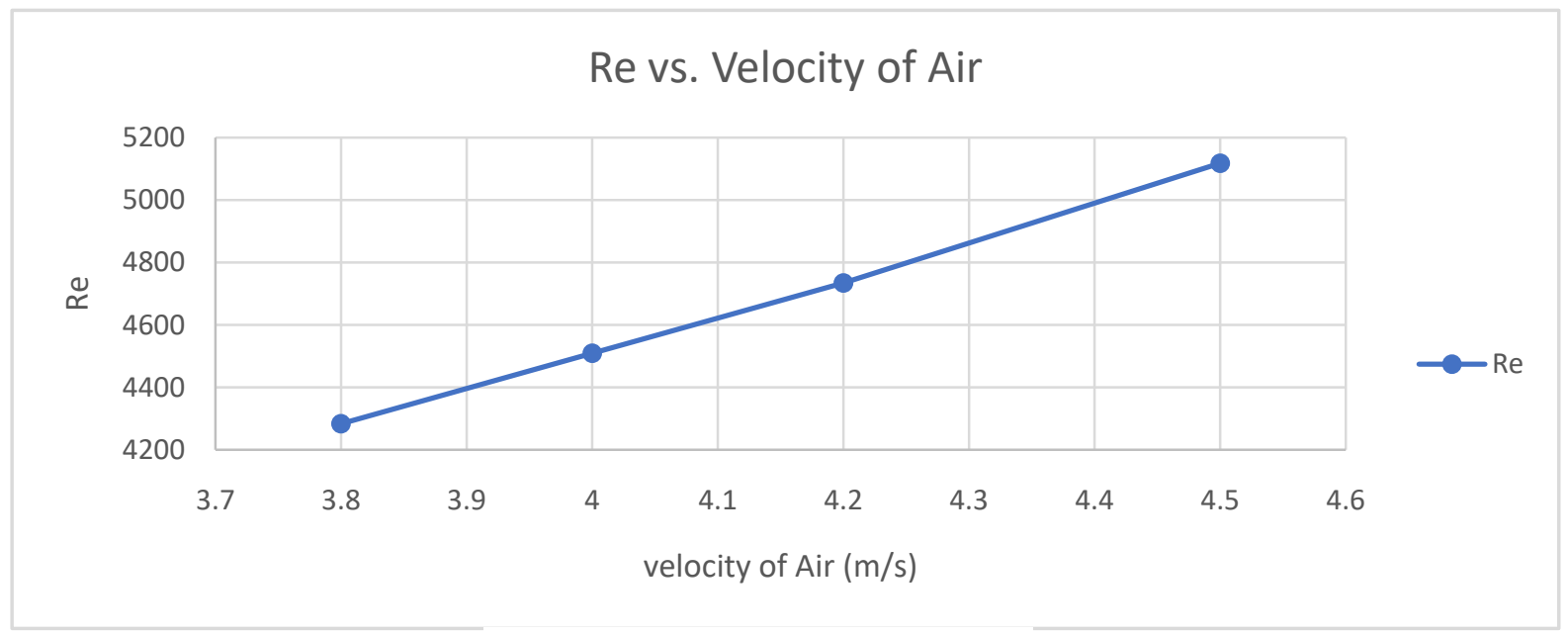

FIG.6Re vs. Air Velocities 


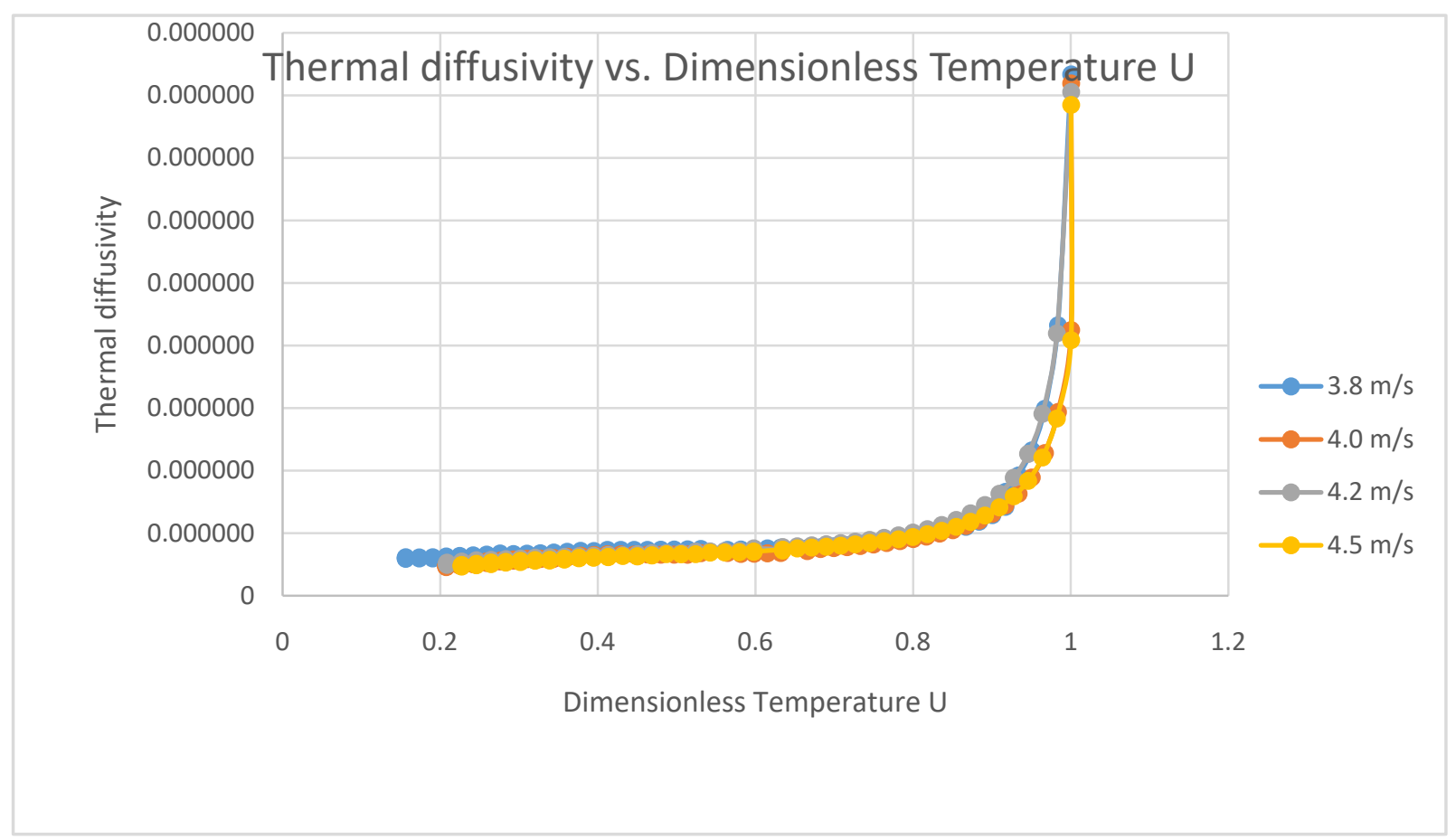

FIG.7 Variation of thermal diffusivity with dimensionless temperature U

The fig. 3 show the variation of temperature with time at 4 different velocities (i.e. 3.8, 4.0, 4.2 \& $4.5 \mathrm{~m} / \mathrm{s}$ ) for cucumber at the center and it is seen that the cooling time increases upto 10-20 minutes for relatively lesser velocity. Fig.4 shows the variation of thermal conductivity with time during cooling of cucumber at the center at four different velocities (i.e. 3.8, 4.0, 4.2 \& 4.5 $\mathrm{m} / \mathrm{s}$ ). As the temperature gradient decreases with time, hence thermal conductivity also decreases with time. For lesser velocity thermal conductivity decreases more. Because for lesser velocities temperature gradient decrement is relatively slower across the cucumber. Fig.5 shows the variation of Nusselt no. and surface film conductance at different Air velocities. It is clearly seen from the graph that with increase in air velocity Nusselt no. and surface film conductance increases. Higher is nusselt number, more will be rate of heat transfer by convection than by conduction. Fig.6 shows the variation of Re with Air velocities, it increases with increase in velocity of air. Fig.7 shows the variation of thermal diffusivity for different air velocities with dimensionless temperature. As we increases temperature thermal diffusivity increases and 
decreases with decreasing temperature. As water is predominant constituents in this food product, the water content of food items significantly influences this property also.

\section{Error Analysis}

Kline-McClintock procedure [23] is used for error analysis. It is used in calculating the dimensionless groups presented in this article.The vulnerability in estimations is characterized as the root entirety square of the fixed blunder by the instrumentation and the irregular mistake saw during various estimations. The mistake examination incorporates estimated temperature. In like manner, deviations in the determined thermal conductivity is $1.8 \%$ from the true value.

\section{Conclusions}

Transient heat transfer behavior during cooling is studied in present work for cylindrical food product (cucumber) at $\mathrm{R}=0$, at different air flow rates.

Temperature measurement is carried out at the center of the specimen at different air flow rates (3.8, 4.0, $4.2 \& 4.5 \mathrm{~m} / \mathrm{s}$ ). These temperature distributions are plotted with time. The terms of Fourier equation were evaluated and thermal diffusivity was plotted against the temperature variation during cooling of cylindrical food product and correlations have been developed for variation of the thermal diffusivity at center of cylindrical food product.

Surface film conductance values is also calculated in present work, at different air flow rates. These values are effective values, which included the additional cooling effect due to moisture evaporation from the exposed product surface.

Variation of Reynolds number, and Nusselt number is also plotted with different air velocities and it is found that more favorable results of thermal conductivity and thermal diffusivity is obtain at $3.8 \mathrm{~m} / \mathrm{s}$. Which ultimately saves energy and provide optimum cooling for the specimen at the given air velocity. 


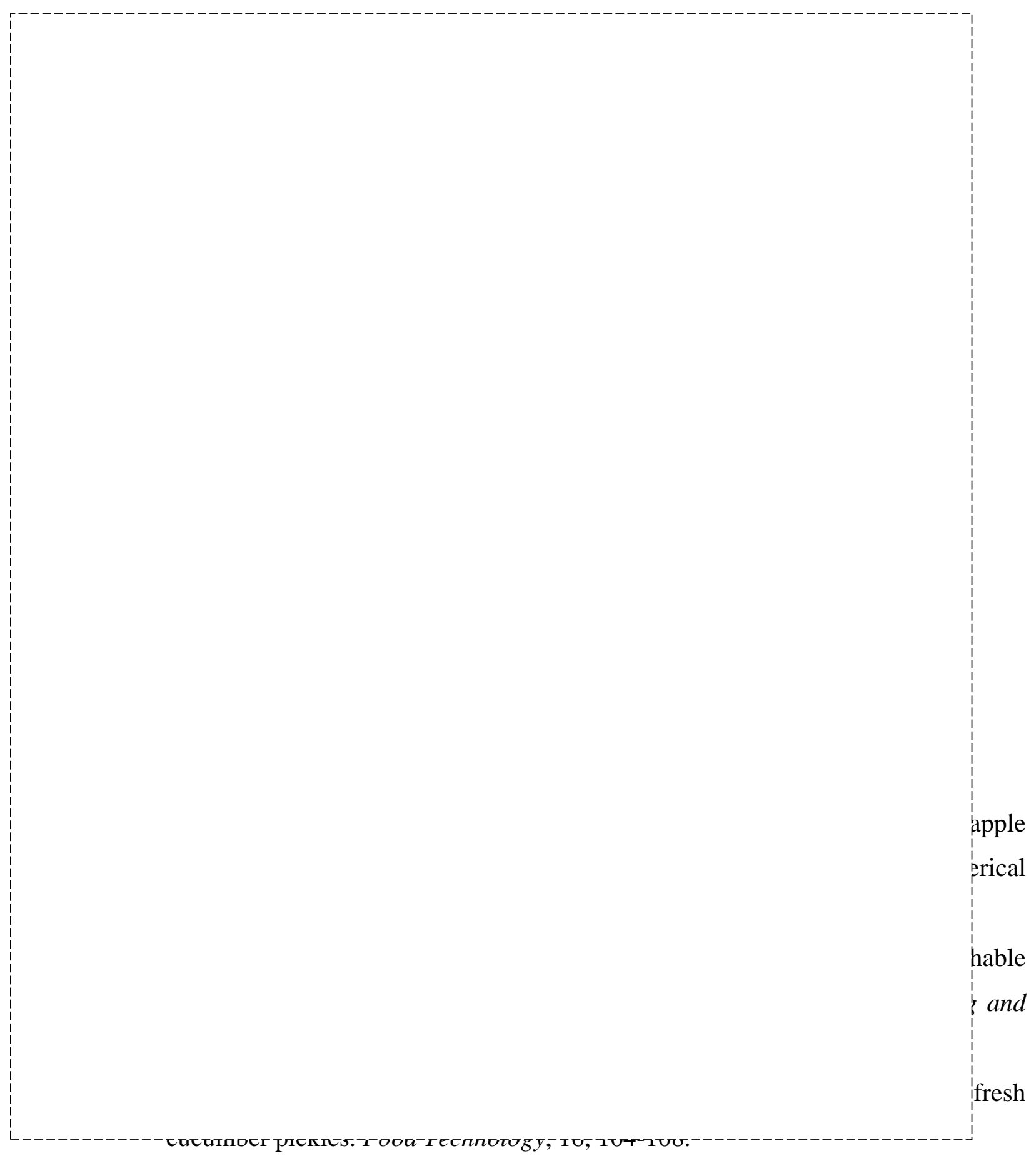

[4] Murthy, S. S., Murthy, M. K., \&Ramachandran, A. (1976). Heat transfer during aircooling and storing of moist food products-II. Spherical and cylindrical shapes. Transactions of the ASAE, 19(3), 577-0583. 
[5] Majeed, P. A. (1982). Prediction of cooling characteristics during air cooling of cylindrical food products with a flowing film of cold water at the surface (air-film cooling). Transactions of the ASAE, 25(2), 508-0514.

[6] De Michelis, A., \&Calvelo, A. (1983). Freezing time predictions for brick and cylindrical-shaped foods. Journal of Food Science, 48(3), 909-913.

[7] Ansari, F. A., \&Afaq, A. (1986). Precooling of cylindrical food products. International Journal of Refrigeration, 9(3), 161-163.

[8] CHUNG, S. L., \& MERRITT, J. H. (1991). Freezing time modeling for small finite cylindrical shaped foodstuff. Journal of Food Science, 56(4), 1072-1075.

[9] Dincer, I., \& Dost, S. (1996). A modelling study for moisture diffusivities and moisture transfer coefficients in drying of solid objects. International Journal of Energy Research, 20(6), 531-539.

[10] Sadashive G B., Narashimhan G S V L and Krishnamurthy M V.(1996). Forced air precooling of spherical food products in bulk-a parametric study. International Journal of Heat \& Fluid Flow, 18, 613-624.

[11] Fasina, O. O., \& Fleming, H. P. (2001). Heat transfer characteristics of cucumbers during blanching. Journal of Food Engineering, 47(3), 203-210.

[12] Brosnan, T., \& Sun, D. W. (2001). Precooling techniques and applications for horticultural products-a review. International Journal of Refrigeration, 24(2), 154-170.

[13] Wu B, Yang W and Jia C.(2004).A three-dimensional numerical simulation of transient heat and mass transfer inside a single rice kernel during the drying process. Biosystems Engineering, 87(2),191-200.

[14] Carmo JEF and Lima AGB.(2005).Drying of lentil including shrinkage: a numerical simulation. Drying Technology, 23(9),1977-1992.

[15] Erdoğdu, F. (2005). Mathematical approaches for use of analytical solutions in experimental determination of heat and mass transfer parameters. Journal of Food Engineering, 68(2), 233-238.

[16] Erdoğdu, F. (2008). A review on simultaneous determination of thermal diffusivity and heat transfer coefficient. Journal of Food Engineering, 86(3), 453459. 
[17] Kumar R, Kumar A, Murthy UN.(2008).Heat transfer during forced air precooling of perishable food products. Biosystems Engineering, 99(2):228-233.

[18] da Silva, W. P., dos Santos Silva, C. M., Nascimento, P. L., Carmo, J. E. D., \& Silva, D. D. P. S. (2011). Influence of the geometry on the numerical simulation of the cooling kinetics of cucumbers. Spanish journal of agricultural research, (1), 242-251.

[19] Da Silva, W. P., \& e Silva, C. M. (2014). Calculation of the convective heat transfer coefficient and thermal diffusivity of cucumbers using numerical simulation and the inverse method. Journal of food science and technology, 51(9), 1750-1761.

[20] ASHRAE, Handbook of Fundamentals(2007).

[21] Gaffeny, J.J., Baird. C.D and Eshleman, W.D.(1980).Review and Analysis of the Transient Method for Determining Thermal Diffusivity of Fruits and Vegetables,” Trans. ASHRAE.

[22] Mascheroni R.H. and Calvelo A.(1980).Relation between heat transfer parameters and the characteristic damage variables for freezing of beef. Meat Science 4 (4), 267-285.

[23] Kline, S. J., and McClintock, F. A. (1953) Describing Uncertainities in Single Sample Experiments, in Mech. Eng. ASME, New York,. 